\title{
Hereditary Spherocytosis Associated with Gilbert Syndrome Diagnosed with Liver Biopsy Examination and Exome Sequencing
}

\author{
Wei Zou1, Zhendong Zhang2, Yongming Tan³ and Lunli Zhang1 \\ Department of Infectious Diseases 1 / Pathology² / Radiology33, The First Affiliated Hospital of Nanchang University, Jiangxi, China
}

\begin{abstract}
Depending on which part of the physiological pathway is affected by the pathology, jaundice is classified into three categories: pre-hepatic/hemolytic, hepatic/hepatocellular, and post-hepatic/cholestatic. With routine laboratory tests, most cases of jaundice can be etiologically diagnosed. However, exceptions do occur. Here, we present a case of a 14-year girl who presented with intermittent jaundice for one year that could not be diagnosed with a routine protocol. Her laboratory tests showed a moderate impairment of liver function and a positive osmotic fragility test. Computed tomography scan of her upper abdomen revealed multiple gallbladder stones and splenomegaly. With the help of liver pathological examination and exome sequencing, this patient was finally diagnosed as hereditary spherocytosis combined with Gilbert syndrome.
\end{abstract}

Key Words: Hereditary spherocytosis, Gilbert syndrome, Liver pathology, Exome sequencing, Spectrin.

How to cite this article: Zou W, Zhang Z, Tan Y, Zhang L. Hereditary spherocytosis associated with Gilbert gyndrome diagnosed wtih liver biopsy examination and exome sequencing. J Coll Physicians Surg Pak 2020; 30(2):213-215.

\section{INTRODUCTION}

Jaundice is a symptom associated with different kinds of diseases. Among them, Gilbert syndrome (GS) is a benign autosomal dominant hereditary disorder characterised by intermittent unconjugated hyperbilirubinemia in the absence of liver disease. ${ }^{1}$ Likewise, hereditary spherocytosis (HS) is a genetic disorder of red blood cell membrane, clinically characterised by anemia, jaundice and splenomegaly. ${ }^{2}$ The calculated rate of coexistence of GS and HS is 15 to 35/million births. ${ }^{3}$ Herein, we present a case with jaundice of unknown cause and finally diagnosed as HS combined with GS by the laboratory test results, imaging findings, and the results of liver pathological examination and exome sequencing.

\section{CASE REPORT}

A 14-year girl presented to our hospital with complain of intermittent yellow sclera, yellow skin and yellow urine accompanied with skin itching and poor appetite for the last one year. She was intermittently treated in a local hospital without significant improvement, and the symptoms aggravated. No special past medical history was noted. After a careful interview with her father, this disease was found to be running in the family of the patient's father. As shown in the pedigree chart of the

Correspondence to: Wei Zou, Department of Infectious Diseases, The First Affiliated Hospital of Nanchang University,

Nanchang, Jiangxi, China, 330006

E-mail: ieeeif@hotmail.com

Received: February 01, 2019; Revised: June 30, 2019;

Accepted: July 10, 2019 family (Figure 1A), besides this patient, her father, grandfather, and possibly her great-grandfather were all affected by this disease that presented with jaundice and hyperbilirubinemia, as explained by the father. Besides yellow skin, yellow sclera and an enlarged spleen, no other abnormal signs were revealed during the physical examination. Liver function panel from the local hospital four days before admission showed alanine transaminase (ALT)/ aspartate transaminase (AST) levels as 488/276 $\mathrm{U} / \mathrm{L}$ and total bilirubin (TB)/direct bilirubin (DB) levels as $278 / 54.4 \mu \mathrm{mol} / \mathrm{L}$. Complete blood count (CBC) showed an elevated white blood cell count of $11.53 \times 10^{\wedge} \mathrm{g} / \mathrm{L}$ and a normal hemoglobin $(\mathrm{Hb})$ level. B-ultrasound of upper abdomen revealed gallbladder stone, cholecystitis, and splenomegaly. Upon admission into our hospital, liver function panel showed a moderate impairment of liver function as indicated by TB/DB level of $96.4 / 8.3 \mu \mathrm{mol} / \mathrm{L}$ and almost normal levels of ALT/AST. CBC showed mild anemia with $\mathrm{Hb}$ of $103 \mathrm{~g} / \mathrm{L}$, a slightly decreased mean corpuscular volume (MCV) of $81.6 \mathrm{fL}$ and normal mean corpuscular hemoglobin concentration (MCHC) (352 g/L). Hepatitis A, B, C and E viruses were all negative. Other hepatotropic viral infections like cytomegaloviral and Epstein-barr viral infection were not evaluated. The antibody panel of autoimmune hepatitis was negative. Thyroid function tests showed an increased serum level of thyroid-stimulating hormone (sTSH) of $5.35 \mu \mathrm{lU} / \mathrm{mL}$ (reference range: $0.27-4.2 \mu \mathrm{lU} / \mathrm{mL}$ ) and normal levels of free tri-iodothyronine (FT3) and free thyroxine (FT4). Blood ceruloplasmin level was slightly decreased (184 $\mathrm{mg} / \mathrm{L}$, reference range: $220-580 \mathrm{mg} / \mathrm{L}$ ). Procalcitonin level was normal. Prothrombin time was 13.9 seconds. Unfortunately, peripheral blood smear and reticulocyte count was not tested. Since indirect bilirubin was much 

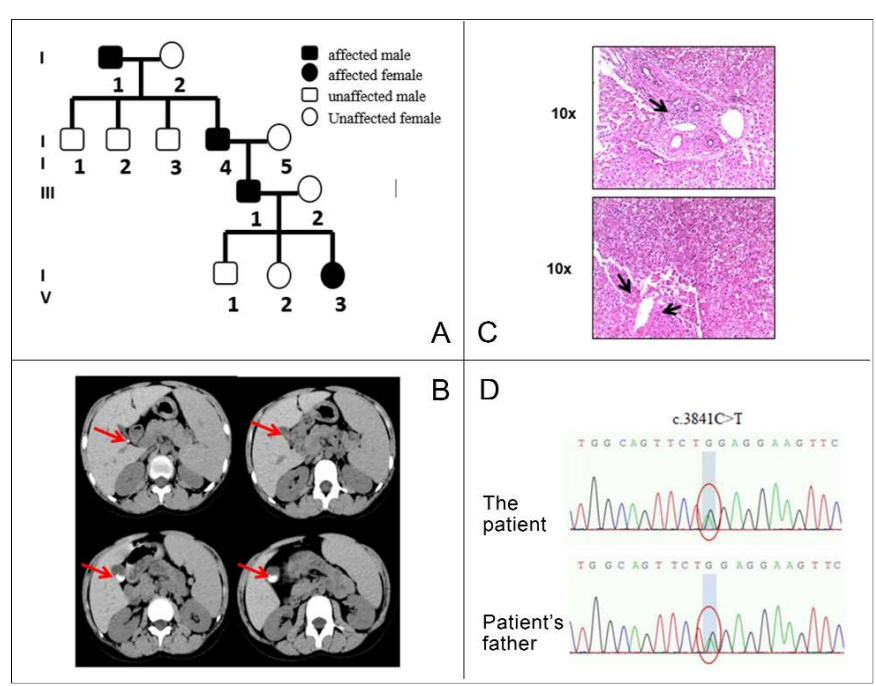

Figure 1: Diagnosis of hereditary spherocytosis together with Gilbert syndrome with genetic, clinical, pathological and exome sequencing data in a teenage girl. (A) The pedigree chart of the disease in the patient's family. (B) CT scan of the upper abdomen of the patient. Red arrows point to the gallbladder stones. (C) Pathology of the liver biopsy sample of the patient. The black arrow in the upper picture indicates the mononuclear cells infiltrating in the sample, and those in the lower picture indicate hepatocytic lipofuscin deposition around the central vein. (D) Exome sequencing plus next generation sequencing of the peripheral blood sample of both, patient and her father, for liver disease-related genes revealed c.3841C > T mutation in the gene of spectrin $\beta$.

more elevated than DB, a panel of hemolysis tests was carried out to determine whether an increased red blood cell (RBC) damage contributed to the jaundice in this patient. This panel of tests included Coomb's test, Ham test, osmotic fragility test and methemoglobin reducing test. Only the osmotic fragility test showed a positive result with a value of $0.52 \%$ at the beginning of hemolysis (reference range: $0.42-0.46$ ) and $0.40 \%$ at the end of hemolysis (reference range: $0.32-0.36$ ). Computed tomography (CT) scan of upper abdomen revealed multiple gallbladder stones and splenomegaly (Figure 1B). To manage this patient's condition, liver protective medications including magnesium isoglycyrrhizinate, polyene phosphatidyl choline, tanshinone, and ornithine aspartate were started after admission. Her blood TB level significantly decreased after one week's treatment. To find out the etiology of her disease, we performed B-ultrasound-guided percutaneous liver biopsy. The pathological examination of liver biopsy sample revealed hepatocellular lipofuscin deposition around the central vein and hepatocellular edema with very few mononuclear inflammatory cells infiltration suggesting GS (Figure 1C). The pathologist also suggested that genome sequencing be performed to confirm the diagnosis. Thus, exome sequencing plus next generation sequencing (Illumina) of the peripheral blood sample of both the patient and her father, was conducted for liver disease-related genes, which have been reported in the literature. The total cost of the exome sequencing for both, patient and her father, was RMB 4500, which is high for most Chinese families. The exome sequencing was performed with the sequence capture human exome microarrays from Agilent Biotechnology Company. The coverage rate and average depth of the target area was $99.3 \%$ and 543 , respectively. $98.9 \%$ of the average depth of the target area was more than 20X. Out of the 170 genes sequenced, a heterozygous mutation in the gene of spectrin $\beta$ (SPTB) on chromosome 14q23 was found with $\mathrm{c} .3841 \mathrm{C}>\mathrm{T}$ mutation, resulting in glutamine being mutated into a stop codon in both, patient and her father (Figure 1D). c.3841C>T mutation has not been reported so far in HGMDpro database. According to American College of Medical Genetics and Genomics (ACMG) gene mutation guidelines, this mutation was classified as a stop mutation (PVS1), no population carrier (PM2), and being likely pathogenic. SPTB mutation leads to cytoskeletal instability and reported to be associated with HS, mostly as autosomal dominant disorder that is caused by RBC membrane defect. So, this patient was finally diagnosed with HS combined with GS, and was discharged from the hospital 11 days after admission.

\section{DISCUSSION}

GS is a common autosomal dominant disorder characterised by intermittent hyperbilirubinemia in the absence of liver disease or hemolysis, mostly as a result of mildly decreased activity of uridine diphosphate-glucuronyl transferase $1 \mathrm{~A} 1$ (UGT1A1). UGT1A $1{ }^{*} 28$, the variant that most commonly causes GS in Caucasian and AfricanAmerican populations, contains seven thymine-adenine (TA) repeats in the promoter region of UGT1A1 gene rather than the typical six, causing impairment of transcription. 4 Although UGT1A1 mutation was not found in the sequencing result of our patient, GS still cannot be excluded as GT1AT1 mutation is not absolutely necessary for the diagnosis of GS, especially considering her medical history, liver pathology result, and hyperbilirubinemia that was out of proportion to the degree of hemolysis. ${ }^{1,4}$

As the most common cause of HS, SPTB defect compromises the ability of erythrocytes to change shape, thus interfering with the cell's ability to be flexibly travelling from the arteries to the smaller capillaries. ${ }^{5}$ This difference in shape makes the RBCs more prone to rupture resulting in inherited hemolytic anemia. 6 This disorder is clinically characterised by fatigue, anemia, jaundice, and splenomegaly with variable severity from symptom-free carrier to severe hemolysis due to incomplete penetrance in its expression. ${ }^{7}$ Common complications include cholelithiasis, hemolytic episodes, and aplastic crises. ${ }^{8}$ Given the family history, symptoms, signs, laboratory tests, and imaging results of this patient, it is reasonable to believe that the $\mathrm{c} .3841 \mathrm{C}>\mathrm{T}$ mutation of SPTB is very likely the etiology of the disease in our patient and her family. Over the follow-up call to her father after her discharge, he said that after discharge from our hospital, she was treated in the local 
hospital for a couple of episodes of recurring jaundice, which is consistent with the clinical characteristics of HS. It is reported that HS may occur in a patient together with GS; and under this circumstance, patients can have jaundice without anemia. In other words, patients can have severe hyperbilirubinemia but little evidence of hemolysis and the resultant anemia, which is consistent with what was found in our patient.

Of critical importance for the diagnosis of GS, individuals should demonstrate elevated bilirubin concentrations two times over a period of six months and should have normal serum transaminases and normal markers of biliary damage/obstruction (gamma-glutamyl transpeptidase and alkaline phosphatase). ${ }^{1} \mathrm{GS}$ is a benign disease and no specific management is necessary; but when it is combined with other risk factors for impairment of liver function, situation may become complicated, which is just what was presented in this case. The liver function panel of this patient from the local hospital four days before admission showed significantly elevated levels of ALT/AST and TB/indirect bilirubin, which is unusual for GS. We speculate that this probably was due to biliary tract infection. In support of this, cholecystitis and elevated white blood cell count $\left(11.53 \times 10^{9}\right)$ was reported from the local hospital. This speculation was further confirmed by the improved laboratory test results from our hospital after admission with normalisation of $\mathrm{CBC}$ accompanied with recovery of liver function.

The massively parallel sequencing technology, known as next-generation sequencing (NGS) has revolutionised biology and medicine. With its ultra-high throughput, scalability, and speed, NGS enables researchers to perform a wide variety of applications and study biological systems at a level never possible before. We believe that with the help of liver pathology examination, NGS and other methods in the diagnosis of the diseases which cannot be diagnosed with traditional methods, a brand-new pattern for the diagnosis and management of difficult liver diseases in the clinic is on the horizon.

\section{PATIENT'S CONSENT:}

Informed consent was obtained from the patient and her father to publish the data concerning this case.

\section{CONFLICT OF INTEREST:}

Authors declared no conflict of interest.

\section{AUTHORS' CONTRIBUTION:}

WZ: Authored the manuscript.

ZZ, YT, LZ: Approved the manuscript.

\section{REFERENCES}

1. Wagner KH, Shiels RG, Lang CA, Seyed Khoei N, Bulmer AC. Diagnostic criteria and contributors to Gilbert's syndrome. Crit Rev Clin Lab Sci 2018; 55:129-39.

2. Bolton-Maggs PH, Langer JC, lolascon A, Tittensor P, King MJ. General haematology task force of the British Committee for Standards in Haematology. Guidelines for the diagnosis and management of hereditary spherocytosis - 2011 update. $\mathrm{Br} \mathrm{J}$ Haematol 2012; 156:37-49.

3. Butorac Ahel I, Baraba Dekanic K, Palcevski G, Roganovic J. An infant with unusually high unconjugated hyper-bilirubinemia due to coexistence of hereditary spherocytosis and Gilbert syndrome. J Pediatr Hematol Oncol 2018; 40: e127-8.

4. Moyer AM, Skierka JM, Kotzer KE, Kluge ML, Black JL, Baudhuin LM. Clinical UGT1A1 genetic analysis in pediatric patients: Experience of a reference laboratory. Mol Diagn Ther 2017; 21:327-35.

5. Maciag M, Plochocka D, Adamowicz-Salach A, Burzynska B. Novel beta spectrin mutations in hereditary spherocytosis associated with decreased levels of mRNA. $\mathrm{Br} J$ Haematol 2009; 146:326-32.

6. Cotran RSK, Fausto V, Nelso N, Robbins F, Stanley L, Abbas AK. Robbins and Cotran pathologic basis of disease 2005; 625.

7. Bolton-Maggs $\mathrm{PH}$, Stevens RF, Dodd NJ, Lamont G, Tittensor $P$, King MJ, et al. Guidelines for the diagnosis and management of hereditary spherocytosis. Br J Haematol 2004; 126:455-74.

8. Perrotta S, Gallagher PG, Mohandas N. Hereditary spherocytosis. Lancet 2008; 372:1411-26. 